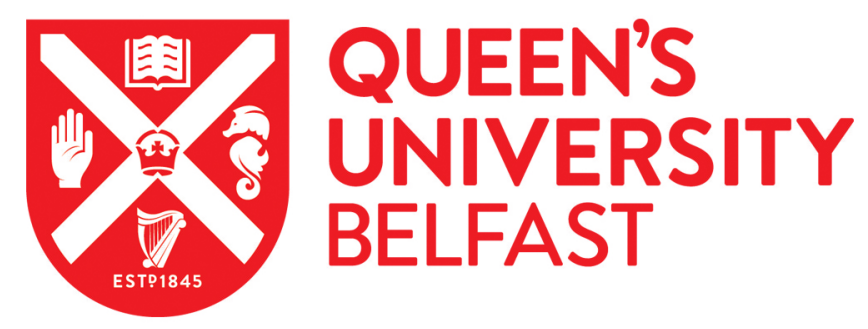

\title{
Above-threshold dissociation of the molecular ion HD+ in a moderate- intensity femtosecond laser field from the calculation of time-of-flight spectra
}

Gao, W., Wang, B-B., Hu, X-J., Chai, S., Han, Y-C., \& Greenwood, J. B. (2017). Above-threshold dissociation of the molecular ion $\mathrm{HD}+$ in a moderate-intensity femtosecond laser field from the calculation of time-of-flight spectra. Physical Review A (Atomic, Molecular, and Optical Physics), 96(1), [013426].

https://doi.org/10.1103/PhysRevA.96.013426

Published in:

Physical Review A (Atomic, Molecular, and Optical Physics)

Document Version:

Peer reviewed version

Queen's University Belfast - Research Portal:

Link to publication record in Queen's University Belfast Research Portal

\section{Publisher rights}

(C2017 American Physical Society.

This work is made available online in accordance with the publisher's policies. Please refer to any applicable terms of use of the publisher.

\section{General rights}

Copyright for the publications made accessible via the Queen's University Belfast Research Portal is retained by the author(s) and / or other copyright owners and it is a condition of accessing these publications that users recognise and abide by the legal requirements associated with these rights.

Take down policy

The Research Portal is Queen's institutional repository that provides access to Queen's research output. Every effort has been made to ensure that content in the Research Portal does not infringe any person's rights, or applicable UK laws. If you discover content in the

Research Portal that you believe breaches copyright or violates any law, please contact openaccess@qub.ac.uk. 


\section{Above threshold dissociation of the molecular ion $\mathrm{HD}^{+}$in moderate-intensity femtosecond laser field from calculation of time of flight spectra}

Wei Gao ${ }^{\dagger}$, Bin-Bin Wang ${ }^{\dagger}$, Xue-Jin $\mathrm{Hu}^{\dagger}$, Shuo Chai ${ }^{\dagger}$, Yong-Chang $\mathrm{Han}^{1 \dagger}$, and J. B. Greenwood

${ }^{\dagger}$ Department of Physics, Dalian University of Technology, Dalian 116024, China

${ }^{\ddagger}$ School of Maths and Physics, Queen's University Belfast, Belfast BT7 1NN, United Kingdom

$2017 / 6 / 22$

${ }^{1}$ Corresponding at Email: ychan@dlut.edu.cn 


\begin{abstract}
The dissociation of the molecular ion $\mathrm{HD}^{+}$in moderate-intensity laser pulses is studied using the time-dependent quantum wave packet method. Simulation of the time of flight (TOF) neutral atom spectra produced from dissociation of the $v=10$ vibrational state at a laser intensity of $10^{12} \mathrm{~W} / \mathrm{cm}^{2}$ and from $v=0$ at the intensity of $5 \times 10^{12} \mathrm{~W} / \mathrm{cm}^{2}$ are calculated. Good agreement is found with previous experimental results [Phys. Rev. Lett. 98, 163001 (2007) and J. Phys. B 42, 154027 (2009)]. Furthermore, the kinetic energy distribution of the dissociated fragments of molecular ion $\mathrm{HD}^{+}$is studied within the moderate laser intensity region from $10^{12}$ to $5 \times 10^{12} \mathrm{~W} / \mathrm{cm}^{2}$ by considering initial vibrational states from $v=0$ to 15 . It is found that the above threshold dissociation (ATD) could be more obviously observed from the TOF spectra when the initial vibrational state is set as $v=6$ for a moderate-intensity femtosecond laser field.
\end{abstract}




\section{INTRODUCTION}

Photodissociation receives considerable attention as a tool for laser control of molecular dynamics [1-4]. Current laser technology produces intense fields, which are comparable with the internal Coulomb field of molecules and activate a variety of mechanisms influencing molecular dissociation (e.g., Refs. 5 and 6). These include above threshold dissociation (ATD) [7,8], bond softening [9-11], vibrational trapping [12,13] and zero photon dissociation $[14,15]$. In the ATD process, more photons are absorbed than are required to overcome the bound energy. The additional quanta of photon energy appear as evenly spread peaks in the kinetic energy spectrum of the atomic photofragment [7].

ATD has long been predicted to be an important dissociation mechanism for a wide range of intensities in a number of theoretical models $[7,8]$. However, there are only a small proportion of dissociation products that possess a higher kinetic energy release as a result of ATD in experiments [16]. The main reason underlying this contradiction between experiment and theory is that the initial vibrational states of the molecular ion could not be selected in the experiments when the neutral molecule is ionized and the daughter ions interact with the laser field within the same laser pulse $[17,18]$. The initial vibrational states of the molecular ion from the multiphoton ionization of neutral molecule have a distribution based upon the Frank-Condon factors [19,20]. The molecular ion will dissociate from distinct initial vibrational states with different energy releases and the corresponding dissociation probabilities also differ by orders of magnitude. Consequently, it is difficult to correlate the spectra with specific vibrational states.

Recently, strong field dissociation of the molecular ion of hydrogen deuteride $\left(\mathrm{HD}^{+}\right)$ was reported experimentally. These ions were produced in an ion source with an initial vibrational state distribution determined by the Franck-Condon factors for ionization from the neutral. The ions were then trapped in an electrostatic ion storage device $[21,22]$ 
for several hundred milliseconds. The heteronuclear molecular ion $\mathrm{HD}^{+}$has a small permanent dipole moment that results in the vibrational energy levels decaying via dipole transitions. The vibrational distribution after a given trapping time can be estimated from theoretical values of the decay rates for each vibrational state [23]. Therefore, the laser pulses could interact with different vibrational distributions of the molecular ion $\mathrm{HD}^{+}$by controlling the trap time. In addition, the TOF technique, which is capable of high resolution kinetic energy analysis of dissociated fragments, is used to study the ATD mechanism in the experiments $[6,21,22]$.

In these papers $[21,22]$, the dissociation mechanisms were investigated through energy analysis of the neutral atoms emitted along the direction of the laser polarization. The flight time of the neutrals to a detector was measured and transformed into the centre of mass to determine the energy release from the dissociation. When the $\mathrm{HD}^{+}$ions were trapped for a short period of time so that there was little vibrational relaxation, the dissociation was dominated by the release energy of $0.8 \mathrm{eV}$ at an intensity of $10^{12} \mathrm{~W} / \mathrm{cm}^{2}$. This corresponded to a resonant 1 photon transition from the $v=10$ vibrational level of the ground electronic state $1 s \sigma_{g}$ to the dissociative first excited state $2 p \sigma_{u}$. For trapping times greater than $0.3 \mathrm{~s}$ this dissociation channel was closed as more than $96 \%$ of the molecules had relaxed into their ground vibrational state $v=0$. In this case, dissociation was dominated by absorption of 2 photons at a laser intensity of $5 \times 10^{12} \mathrm{~W} / \mathrm{cm}^{2}$, but there was a small contribution from ATD at higher release energies corresponding to absorptions of 3,4 or 5 photons. When the intensity was increased to a peak of $10^{15}$ $\mathrm{W} / \mathrm{cm}^{2}$, this ATD process was the dominant dissociation mechanism.

Substantial effort has been devoted to theoretical studies of the dissociation of the $\mathrm{HD}^{+}$molecular ion [24-31]. For instance, the kinetic energy spectra for the dissociation of this system at high intensities $\left(10^{14} \sim 10^{15} \mathrm{~W} / \mathrm{cm}^{2}\right)$ were calculated by using the wavepacket method [24,26], which supported the experimental findings that absorption of 4 or 5 photons was occurring at high intensities. Besides the ATD dynamics, many 
other issues were also investigated, such as the control of the asymmetry branching ratio of the photofragments [28], the interferences between dissociative wavepackets [29], the preparation and probing of the coherent vibrational wavepacket [30], the steering of the electron localization [31], etc. Most of these studies were based on the calculation of the kinetic energy distribution of dissociated fragments, and to the best of our knowledge, the ATD process of the $\mathrm{HD}^{+}$molecular ion has not yet been thoroughly and quantitatively explained in a theory-versus-experiment comparison via a TOF spectrum. In addition, most of the studies of the ATD process were concerned about the interaction of the molecule with strong laser fields $\left(\geqslant 10^{13} \mathrm{~W} / \mathrm{cm}^{2}\right)$, while the ATD signal was assumed to be negligible at lower laser intensities. As mentioned above, in Ref. [21], one can observe the ATD signal at a strong intensity of $10^{15} \mathrm{~W} / \mathrm{cm}^{2}$, but the ATD signal at a moderate intensity of $5 \times 10^{12} \mathrm{~W} / \mathrm{cm}^{2}$ could not be clearly identified from the TOF spectrum.

We also note that in the strong-field regime, many phenomena related to the electronic dynamics may occur, including the ionization, recombination, electronic-state coupling, etc., and these processes can influence the nuclear dynamics. For example, high intensities can induce enhanced ionization at large nuclear distances due to electron localization [9,32]; high intensities can also result in the laser induced conical intersections (LICIs), which can be characterized by quantum interference, i.e., modulations in the angular distributions of the dissociated fragments [33,34]. In the strong-field regime, the nuclear and electronic dynamics usually interact or correlate with each other (see, e.g., Refs. [35-39]).

Thus, at moderate laser intensities, where the correlations between electronic and the nuclear dynamics are negligible, it is an open question whether one could observe significant ATD signals via the TOF spectrum technique. To answer this question we first reproduce the previous experimental measurements of the TOF spectrum for the $\mathrm{HD}^{+}$ molecular ion. Calculations are made under two different conditions corresponding to the TOF spectrum in the laboratory frame at an intensity of $10^{12} \mathrm{~W} / \mathrm{cm}^{2}$ and the TOF 
spectrum in the center-of-mass frame at $5 \times 10^{12} \mathrm{~W} / \mathrm{cm}^{2}$. Based on the good agreement with experiments, we then performed further studies to find a TOF-spectrum-observable ATD signal by considering the dissociation from the vibrational states from $v=0$ to $v=$ 15 within this moderate intensity region from $10^{12}$ to $5 \times 10^{12} \mathrm{~W} / \mathrm{cm}^{2}$. It is found that the ATD signal can be clearly observed from the TOF spectrum for dissociation from the $v=$ 6 state in this moderate laser field.

The paper is organized as follows. In Sec. II, we describe the theoretical method briefly. The results are presented and discussed in detail in Sec. III, and in Sec. IV, the conclusions are summarized.

\section{THEORETICAL TREATMENTS}

Our numerical calculations include the two lowest electronic states of the molecular ion $\mathrm{HD}^{+}$, the attractive ground ${ }^{2} \Sigma_{\mathrm{g}}^{+}\left(1 s \sigma_{\mathrm{g}}\right)$ and repulsive excited ${ }^{2} \Sigma_{\mathrm{u}}^{+}\left(2 p \sigma_{\mathrm{u}}\right)$ states [40]. In the adiabatic representation, the time-dependent Schrödinger equation (TDSE) beyond the Born-Oppenheimer approximation describing the interaction of molecular ions with an external laser field can be written as [41]

$$
\begin{aligned}
i \frac{\partial}{\partial t}\left(\begin{array}{l}
F_{1}(R, t) \\
F_{2}(R, t)
\end{array}\right)= & -\frac{1}{2 \mu}\left\{\left(\begin{array}{cc}
\partial / \partial R & 0 \\
0 & \partial / \partial R
\end{array}\right)+\left(\begin{array}{cc}
0 & P_{12}(R) \\
P_{12}(R) & 0
\end{array}\right)\right\}^{2}\left(\begin{array}{l}
F_{1}(R, t) \\
F_{2}(R, t)
\end{array}\right) \\
& +\left(\begin{array}{cc}
V_{1}(R)+\Delta E_{11}(R) & 0 \\
0 & V_{2}(R)+\Delta E_{22}(R)
\end{array}\right)\left(\begin{array}{l}
F_{1}(R, t) \\
F_{2}(R, t)
\end{array}\right) \\
& -\mathcal{E}(t)\left(\begin{array}{ll}
d_{11}(R) & d_{12}(R) \\
d_{21}(R) & d_{22}(R)
\end{array}\right)\left(\begin{array}{l}
F_{1}(R, t) \\
F_{2}(R, t)
\end{array}\right)
\end{aligned}
$$

with

$$
\begin{aligned}
& \Delta E_{11}(R) \approx-\frac{1}{2 \mu}\left[Q_{11}(R)-P_{12}(R)^{2}\right], \\
& \Delta E_{22}(R) \approx-\frac{1}{2 \mu}\left[Q_{22}(R)-P_{12}(R)^{2}\right] .
\end{aligned}
$$


Here, $\mu$ is the reduced mass of the molecular ion $\mathrm{HD}^{+}, R$ is the inter-nuclear distance, and $V_{i}(R)$ with $i=1,2$ is the adiabatic potential energy curve of the two lowest electronic states (i.e., $1 s \sigma_{\mathrm{g}}$ and $2 p \sigma_{\mathrm{u}}$ ), respectively. $d_{12}(R)=d_{21}(R)$ denotes the transition dipole moment between the $1 s \sigma_{\mathrm{g}}$ and $2 p \sigma_{\mathrm{u}}$ states. Terms $d_{11}(R)$ and $d_{22}(R)$ denote the permanent dipole moment of $1 s \sigma_{\mathrm{g}}$ and $2 p \sigma_{\mathrm{u}}$ states, respectively. The $P_{i j}(R)$ and $Q_{i j}(R)$ represent the nonadiabatic effects resulting from the action of the nuclear derivatives on the channel functions. The relevant adiabatic potential energy curves, and the nonadiabatic couplings and dipole moment for the molecular ion $\mathrm{HD}^{+}$are illustrated in Fig. 1. The laser field $\varepsilon(t)$ can be written as

$$
\begin{aligned}
\varepsilon(t)=f(t) & \cos \left[\omega\left(t-t_{0}\right)\right] \\
= & \varepsilon_{0} \exp \left[-4 \ln 2\left(\frac{t-t_{0}}{\tau_{\mathrm{p}}}\right)\right] \cos \left[\omega\left(t-t_{0}\right)\right],
\end{aligned}
$$

where $f(t)$ denotes the envelope of electric field amplitude, and $\varepsilon_{0}, t_{0}, \tau_{p}, \omega$ are the peak amplitude, central time, full width at half maximum (FWHM), and the central angular frequency, respectively. For the peak amplitude $\varepsilon_{0}$, the laser intensity is $I_{0}=0.5 \mathrm{ce}_{0} \varepsilon_{0}{ }^{2}$, where $\mathrm{c}$ is the velocity of the light and $\mathrm{e}_{0}$ is the dielectric constant.

Eq. (1) is evolved using the adiabatic-diabatic transformation with the split-operator method [41]. The initial wave function of the molecular ion $\mathrm{HD}^{+}$is obtained by using the Fourier-grid-Hamiltonian (FGH) method [42]. Actually, we could solve the full-dimensional TDSE (including the molecular rotational freedom) for the titled process. However, in the experiment we aimed to simulate the finite acceptance solid angle of the channel electron multiplier (CEM) detector, which means that only dissociation along the polarization direction of the laser pulse could be observed [21,22]. Thus, in this work, to be consistent with the experiment, we have to limited our treatment to solving the one-dimensional $(J=0)$ TDSE. Nevertheless, the nonadiabatic coupling beyond the Born-Oppenheimer approximation has to be taken into consideration [see Eqs. (1) and (2)] to complete the theory. 
To identify the multiphoton dissociation process, it is very useful to calculate the kinetic energy distribution of the dissociated fragments. At a proper time point, when the dissociating wave packets have completely passed the diabatic coupling region (around roughly 12 Bohr), the kinetic energy distribution of dissociated fragments is obtained by projecting the time-dependent wave function onto the continuum states [43].

$$
\rho\left(E_{K}\right)=\left|\left\langle\varphi\left(E_{K}, R\right) \mid F_{i}\left(R, t_{0}\right)\right\rangle\right|^{2} \quad(i=1,2) .
$$

Here, $\varphi\left(E_{k}, R\right)$ are continuum states for a given energy $E_{k}$, and $F_{i}\left(R, t_{0}\right)$ denotes the time-dependent wave function $F_{i}(R, t)$ at time point $t_{0}$. We also note that $E_{k}$ is the relative kinetic energy between dissociating fragments in the center-of-mass frame.

To compare our calculations with the experimental results, the kinetic energy distribution of dissociated fragments needs to be converted to a TOF spectrum of the dissociated fragments. The $\mathrm{HD}^{+}$beam from the ion source possesses 1 and $2 \mathrm{keV}$ kinetic energies $E$ along the axis [21,22]. Although the laboratory keV kinetic energies $E$ are much greater than the $\mathrm{eV}$ kinetic energy release in the center-of-mass frame, a velocity transformation to the laboratory frame readily shows that it is possible to distinguish between different dissociation processes from the neutrals' TOF to the channel electron multiplier (CEM) detector. In the laboratory frame, the half-angle acceptance of the CEM is small. Thus, only those fragments that have small velocity components perpendicular to the axis of the detector are considered in the theoretical calculations.

Each neutral fragment can be emitted in two directions, parallel or anti-parallel to the CEM detector. In laboratory coordinates, the TOF of neutral dissociated fragments is expressed as [22]

$$
T_{H / D}^{ \pm} \approx \frac{L}{v}\left\{1 \pm\left[\frac{\left(m-m_{H / D}\right) E_{K}}{m_{H / D} E}\right]^{1 / 2}\right\},
$$

where the superscripts \pm correspond to the backward $(+)$ and forward (-) neutral fragments, respectively. Here, $m$ is the mass of $\mathrm{HD}^{+}$, and $m_{H / D}$ is mass of neutral 
dissociated fragment. $E_{k}$ is the energy of the dissociated fragments in the center-of-mass frame. $L$ is the distance between the interaction point and the CEM $(L=0.727 \mathrm{~m})$ [44]. $E$ is the initial kinetic energy of $\mathrm{HD}^{+}$from the ion source, and $v$ is the initial velocity of the ion beam. Therefore, the relative kinetic energy distribution from Eq. (4) can be converted to the TOF spectrum $\rho_{\mathrm{H} / \mathrm{D}}(T, E)$ via Eq. (5). In other words, given $E$, the initial kinetic energy of the $\mathrm{HD}^{+}$ion source, we can obtain $\rho\left(T^{+}, E\right)$ and $\rho(T, E)$ from $\rho\left(E_{k}\right)$ for either the $\mathrm{H}$ or the $\mathrm{D}$ fragment.

Nevertheless, it is worth noting that there is an energy spread (full width half maximum) $\delta E$ produced from the ion source in the experiment. The energy spread for a 1 $\mathrm{keV} \mathrm{HD}$ beam is about $\delta E=30 \mathrm{eV}$, and for a $2 \mathrm{keV} \mathrm{HD} \mathrm{HD}^{+}$beam it is about $\delta E=60 \mathrm{eV}$. Thus, in the numerical calculations, the initial energy of ion beam is considered to be a Gaussian-like distribution that is written as

$$
f(E)=\frac{1}{\sqrt{2 \pi} \sigma} \exp \left(-\frac{\left(E-E_{0}\right)^{2}}{2 \sigma^{2}}\right),
$$

with an energy spread

$$
\delta E=2 \sigma \sqrt{2 \ln 2}
$$

where $E$ denotes the initial energy of ion beam, $E_{0}$ is center energy of ion beam, $E_{0}=1$ $\mathrm{keV}$ for $\delta E=30 \mathrm{eV}$ and $E_{0}=2 \mathrm{keV}$ for $\delta E=60 \mathrm{eV}$, respectively.

Thus, the TOF spectrum of the dissociated fragments can be written as

$$
\rho_{H / D}\left(T^{ \pm}\right)=\int \rho_{H / D}\left(T^{ \pm}, E\right) f(E) d E .
$$

\section{RESULTS AND DISCUSSIONS}

We first calculate the TOF spectra of the neutral H and D fragments. These spectra are also useful in detecting the dissociation signal of the molecular ion $\mathrm{HD}^{+}$in experiments $[21,22]$. The laser parameters in the numerical simulations are consistent with the experimental values and set to $I_{0}=10^{12} \mathrm{~W} / \mathrm{cm}^{2}$ ( or $5 \times 10^{12} \mathrm{~W} / \mathrm{cm}^{2}$ ), $\tau_{p}=40$ fs and 
$\omega=12,509 \mathrm{~cm}^{-1}$ (corresponding to $800 \mathrm{~nm}$ wavelength). In the moderate-intensity laser field, the molecular ion $\mathrm{HD}^{+}$is mainly dissociated by two different channels: $1 s \sigma_{\mathrm{g}}$ for $\mathrm{H}^{+}+\mathrm{D}$ and $2 p \sigma_{u}$ for $\mathrm{H}+\mathrm{D}^{+}$.

With a laser intensity $I_{0}=10^{12} \mathrm{~W} / \mathrm{cm}^{2}$, the TOF spectrum from the vibrational state $v$ $=10$ is obtained in the laboratory coordinates from dissociation of $1 \mathrm{keV} \mathrm{HD}{ }^{+}$ions. Figure 2 shows that there are four distinct peaks - two large peaks from the $\mathrm{H}$ fragments with two small shoulders from the D fragments. This indicates that dissociation from the channel $2 p \sigma_{u}\left(\mathrm{H}^{+} \mathrm{D}^{+}\right)$is more probable than that from the channel $1 s \sigma_{\mathrm{g}}\left(\mathrm{H}^{+}+\mathrm{D}\right)$. This theoretical work is used to prepare a TOF spectrum via Eq. (8). The tiny energy spacing of numerical integration $d E$ also leads to the different $d T$ values between the forward and backward fragments in Eq. (5). This is why the forward peak is larger than the backward peak for both $\mathrm{H}$ and $\mathrm{D}$ fragments. The peak separations indicate that the initial vibrational state $v=10$ crosses with the 1-photon-dressed $2 p \sigma_{u}$ state and results in $\mathrm{HD}^{+}$dissociation with a release of kinetic energy $0.8 \mathrm{eV}$.

Figure 2 could not be directly compared with experiment, because in experiment, the detection efficiency of $\mathrm{H}$ fragments is much lower than that of $\mathrm{D}$ fragments since the former has only half of the energy of the latter [22]. Thus, we renormalize the relative ratio of the $\mathrm{H}$ fragments to the $\mathrm{D}$ fragments according to the experimental detection efficiency and the result is shown in Fig. 3. This renormalization factor is also applied to the following theoretical calculations of TOF spectra. In Fig. 3, the TOF spectrum has been shifted down by $50 \mathrm{~ns}$ compared to the experimental result. This is because the zero energy release point is difficult to ascertain from the numerical calculations.

Figure 3 gives a direct comparison between the experimental and theoretical results. The agreement is good, but there are still some differences since there are a range of vibrational states populated in the experiment [44] while the initial vibrational state is held constant at $v=10$ in the theoretical calculations. In addition, the rotational degree of freedom is neglected in our work due to the small half-angle acceptance of the CEM. 
To improve the statistics of the previous experimental data, the TOF spectrum of neutral dissociated fragments was converted to the center-of-mass frame [21]. Thus, we calculate the TOF spectrum in the center-of-mass frame, considering the dissociation from ground vibrational level $(v=0)$ by a $5 \times 10^{12} \mathrm{~W} / \mathrm{cm}^{2}$ laser pulse. Figure 4 shows the theoretical calculations compared with the experimental results in the TOF center-of-mass frame. The peaks of the TOF spectrum in both theory and experiment are consistent with the absorption of 2 photons. This indicates that dissociation from the ground vibrational state mainly occurs via a 2 photon dissociation process. 2 photons are the minimum required for dissociation from the ground vibrational level, and hence ATD only occurs if the dissociation wave packets absorb excess photons. Thus, the ATD signal is barely visible in Fig. 4, although the $\mathrm{H}$ and $\mathrm{D}$ fragments, which possess identical TOF, are summed to improve statistics. Moreover, the distinctions between the theoretical calculations and the experimental results in Fig. 4 may stem from the fact that in experiment, the ion energy spread reduces the resolution of potential ATD channels [21].

In the following discussion, we refer to the kinetic energy distribution of the dissociated fragments to indicate that there are actually tiny ATD signals from the initial vibrational state $v=0$ with the laser intensity at $I_{0}=5 \times 10^{12} \mathrm{~W} / \mathrm{cm}^{2}$. To directly observe the probability of absorbing different numbers of photons, we performed a transformation of the kinetic energy distribution of dissociated fragments (Fig. 5). The horizontal axis indicates the energy difference between dissociated fragments and the initial vibrational state $(v=0)$. Because the probability of dissociation from the absorption of different numbers of photons may differ by several orders of magnitude, the yield is plotted on a logarithmic scale. Figure 5 shows that the branching ratios of dissociated fragments between the two channels are nearly identical. It also indicates that the 2 photon dissociation probability is nearly two orders of magnitude larger than that of 3,4 , or 5 photons. Thus, ATD process from the initial vibrational state $v=0$ is likely to lie under the tail of the 2 photon signal in the experimental TOF spectrum. 
The initial vibrational levels and laser intensity clearly play important roles in the ATD process $[8,21,30]$. Here, we expand the discussion by calculating the kinetic energy distribution of dissociated fragments at a variety of laser intensities and a wide range of initial vibrational states $(v=0-15) .2$ photons are the minimum required for dissociation from lower vibrational states (i.e., $v \leq 5$ ), while the molecular ion $\mathrm{HD}^{+}$can be dissociated from higher vibrational states (i.e., $v>5$ ) by net absorption of a single photon. As seen in Fig. 6, the ATD process occurs in a number of different initial vibrational states for a range of laser intensities. It is also evident that the branching ratios of the two dissociation channels are very similar, with the ratio of H/D production nearly 1 at higher laser intensities [24]. We also note that as $I_{0}$ and $v$ increase, the dissociation probability from absorption of single photon and multiple photons gradually increases. The ATD signal is almost invisible for initial vibrational states corresponding to $v \leq 5$. By contrast, although the probability of ATD from the initial vibrational state $(v \geq 7)$ is relatively large, the dissociation signal from absorption of a single photon would completely dominate the ATD signal in an experiment. However, a strong ATD signal without interference from the single photon channel should be clearly observed when the $v=6$ vibrational state is populated, for a range of laser intensities.

Figure 7(a) shows theoretical simulations of the neutral product TOF spectrum in the laboratory frame for $\mathrm{HD}^{+}$ions in the $v=6$ vibrational state. The separation of the peaks arises from a $1.68 \mathrm{eV}$ energy release from absorption of two $800 \mathrm{~nm}$ photons. The small shoulders correspond to the absorption of 3 photons. In addition, the TOF spectra in the center-of-mass frame for neutral $\mathrm{H}$ and $\mathrm{D}$ fragments are shown in Fig. 7(b). It can be seen that the largest peaks of $\mathrm{D}$ and $\mathrm{H}$ are due to the energy release resulting from absorption of 2 photons. A vague D shoulder at around 65-75 ns and a small $\mathrm{H}$ peak at around 115-125 ns indicate the absorption of 3 photons. Therefore, we show that significant ATD signal from $v=6$ can be obtained at $I_{0}=5 \times 10^{12} \mathrm{~W} / \mathrm{cm}^{2}$ from the TOF spectrum of neutral dissociated fragments. We have checked that at an even lower laser intensity of $I_{0}$ 
$=10^{12} \mathrm{~W} / \mathrm{cm}^{2}$, such an ATD signal can still be observed from the TOF spectrum.

The dissociation process for absorbing different photon numbers can be easily understood from the dressed-states representation (see, e.g., Refs. [7,8,21,24,27,30]). This light field-molecule system can be represented by the field-free potential energy curves of the ground state $1 s \sigma_{g}$ and excited state $2 p \sigma_{u}$, dressed by a number of photons, with states separated by 1 photon energy. As shown in Fig. 8 , in this representation, a series of avoided crossings in the adiabatic limit can be formed due to the strong mixing of the two states, when the laser intensity increases.

At moderate laser intensities, the field-free ground state $1 s \sigma_{g}$ can be coupled to the $2 p \sigma_{u}$ state dressed by $1,2,3$ and 4 photons, as marked by the blue squares labeled by a, b, $\mathrm{c}$, and $\mathrm{d}$ in Fig. 8. The corresponding coupling strengths decrease as the number of dressing photons increases. The couplings induced by even more photons (i.e., 5, and 6 photons) can be neglected for this laser intensity. For comparison, we also marked the energy levels for the $v=0,6$, and 10 states in the field-free ground state $1 s \sigma_{g}$. Thus, it can be understood why the ATD signal is more likely to be observed starting from $v=6$ than starting from $v=0$ or 10 . The $v=0$ state is too deeply bound to present strong ATD signals, and the dissociation would weakly occur via the absorption of 2 photons, the minimum required, and the contributions from ATD at higher release energies corresponding to absorption of 3,4 or 5 photons are even smaller. By contrast, dissociation of the higher energy $v=10$ state is dominated by direct dissociation via the 1 photon process.

The photodissociation from $v=6$ is much more accessible for the ATD process via 2 or 3 photons. The 1 photon dissociation from the $v=6$ state can occur either by tunnelling through the barrier around the 1-photon-dressed crossing point a or by absorbing 2 photons followed by 1 photon reemission around the crossing point $\mathrm{N}$. The former path produces the fragments $\mathrm{D}^{+}+\mathrm{H}$ from the excited state $2 p \sigma_{u}$, while the latter produces $\mathrm{D}+\mathrm{H}^{+}$from the ground state $1 s \sigma_{g}$. It is straightforward to understand that the tunnelling rate for the former path is rather small for such a broad and high barrier. The probability for the latter path is also weak, because of the effect of vibrational trapping $[12,13]$. When the 2-photon gap is open around the crossing point $b$, part of the vibrational wavepacket would be trapped in the laser-induced well above it, because the 
initial $v=6$ state is approximately at the same level as the vibrational ground state in the laser-induced well. On the other hand, the ATD dissociation channels involving net absorption of 2 or 3 photons are wide open for the $v=6$ state. Thus, starting from $v=6$, distributions from the 2 and 3 photon ATD signals are much greater than that from the 1 photon dissociation signal.

Note that in the above results and discussions, the nonadiabatic couplings beyond the Born-Oppenheimer (BO) approximation have been taken into consideration in overall computational procedures. From hereon we refer to these as the non-BO results. The nonadiabatic couplings, which are relatively strong around the internuclear distance $R=$ 12 a.u., can cause redistribution of the wavepackets between the ground and excited electronic states [41]. To distinguish the role of the nonadiabatic couplings on the dissociation dynamics, we further performed the calculations with the nonadiabatic couplings turned off, i.e., artificially setting $P_{i j}$ and $Q_{i j}$ in Eq. (1) to be zero. For convenience, we refer to these results as the $\mathrm{BO}$ results.

The kinetic energy distributions of dissociation fragments with the nonadiabatic couplings turned off are shown in Fig. 9. These can be compared with Fig. 6, in which the couplings are taken into account. We notice that the general trends for the kinetic energy distributions varying with the laser intensity and the initial vibrational state are not obviously changed after we turned off the nonadiabatic couplings. However, for given kinetic energies, the dissociation probabilities from the ground and excited states are obviously different in Fig. 9. For instance, for the kinetic energy corresponding to 2-photon dissociation from $v=3 \sim 8$, the dissociation probability from the ground state in Fig. 9(e) is much higher than that from the excited state in Fig. 9(i). This is different from the non-BO results in Fig. 6, where the dissociation probabilities from the ground and excited states are almost the same. This indicates that before the dissociation wavepackets enter the nonadiabatic coupling region around $R=12$ a.u., the dissociation probabilities from the two electronic states are actually different due to various dissociation mechanisms, and the nonadiabatic coupling then redistributes the branching ratios between the two electronic states.

Specifically, we consider the case of an initial state $v=6$ at the laser intensity $I_{0}=5$ $\times 10^{12} \mathrm{~W} / \mathrm{cm}^{2}$, to compare the kinetic energy distributions in Fig. 10 and the TOF spectra 
in Fig. 11 between the $\mathrm{BO}$ and non-BO results. As mentioned above, in the $\mathrm{BO}$ results as shown in Fig. 10(a), the dissociation probabilities from the two electronic states are obviously different, for instance, the magnitude of the 2-photon dissociation probability from the ground state is roughly one-order greater than that from the excited state, and the magnitude of the 3-photon dissociation probability from the ground state is roughly one-order smaller than that from the excited state.

The difference in the branching ratios between the two electronic states in the $\mathrm{BO}$ results can be related to the variation of the dissociation paths. In the $\mathrm{BO}$ results, to achieve 2-photon dissociation from the ground state, the nuclear wavepacket from the $v=$ 6 state first passes the 3-photon gap around crossing point $\mathrm{c}$, and then reemits 1 photon around crossing point $\mathrm{M}$, as marked in Fig. 8; while to achieve 2-photon dissociation from the excited state, the wavepacket has to pass the 2-photon gap directly without reemitting a photon, which has been explained to be rather weak because of vibrational trapping. Thus, in the $\mathrm{BO}$ results, the 2-photon dissociation probability from the ground state is relatively higher than that from the excited state. It is different for the dissociation by net absorption of 3 photons. To dissociate from the ground state, the wavepacket first passes the 4-photon gap around crossing point $\mathrm{d}$, and then reemits 1 photon around point Q; while to dissociate from the excited state, the wavepacket can pass the 3-photon gap without reemission. Obviously, the 3-photon-dressed gap is much more likely to be open than the 4-photon-dressed gap, which is consistent with the findings in Fig. 10(a) that the 3-photon dissociation probability from the excited state is greater than that from the ground state.

Due to the redistribution effect by the nonadiabatic couplings, the dissociation probabilities from the two electronic states become identical, as the non-BO results show in Fig. 10(b). Additionally, the TOF spectra from the BO and non-BO calculations are compared in Fig. 11. In the BO calculations, one can only observe 2-photon dissociation on the $\mathrm{D}+\mathrm{H}^{+}$channel and 3-photon dissociation from the $\mathrm{H}+\mathrm{D}^{+}$channel; nevertheless, in the non-BO calculations, 2- and 3-photon dissociations from both channels are observable. This is consistent with the kinetic energy distribution shown in Fig. 10. Thus, the calculations beyond the $\mathrm{BO}$ approximation accurately model the TOF spectrum which provides a benchmark for future experiments. 


\section{CONCLUSION}

In this paper, we have investigated the ATD of the molecular ion $\mathrm{HD}^{+}$at moderate laser intensities by solving the time-dependent Schrödinger equation beyond the Born-Oppenheimer approximation. We focused on the question addressed at the beginning of this paper, whether one could observe significant ATD signals in moderate-intensity laser fields via dissociation from an ion beam using the TOF technique.

Firstly, we obtained accurate TOF spectra which are in good agreement with the experimental observations in both the laboratory and the center-of-mass frames at two moderate laser intensities, $10^{12}$ and $5 \times 10^{12} \mathrm{~W} / \mathrm{cm}^{2}$, for dissociation from the $v=10$ and $v$ $=0$ vibrational states respectively. We further calculated the kinetic energy distribution of dissociated fragments for a wide range of initial vibrational states $(v=0-15)$ in this moderate laser intensity region from $10^{12}$ to $5 \times 10^{12} \mathrm{~W} / \mathrm{cm}^{2}$. It is found that by properly choosing the initial vibrational state, one can observe significant ATD signal from the TOF spectra at moderate laser intensities. For instance, it could be clearly observed from the TOF spectra that the ATD processes via the absorption of 2 and 3 photons are the dominant dissociation mechanism, if the initial vibrational state is set to be $v=6$ at moderate laser intensities $\left(10^{12} \sim 5 \times 10^{12} \mathrm{~W} / \mathrm{cm}^{2}\right)$.

\section{ACKNOWLEDGMENT}

The project is supported by the National Natural Science Foundation of China under Grant No. 21473018; the Liaoning BaiQianWan Talents Program; the Fundamental Research Funds for the Central Universities [DUT15YQ105]; The Natural Science Foundation of Liaoning Province (201602146). 


\section{References:}

[1] M. Dantus, Annu. Rev. Phys. Chem. 52, 639 (2001).

[2] K. Sändig, H. Figger, and T. W. Hänsch, Phys. Rev. Lett. 85, 4876 (2000).

[3] H. Niikura, F. Légaré, R. Hasbani, M. Y. Ivanov, D. M. Villeneuve, and P. B. Corkum, Nature 421, $826(2003)$.

[4] X. M. Tong, Z. X. Zhao, and C. D. Lin, Phys. Rev. Lett. 91, 233203 (2003).

[5] A. Giusti-Suzor, F. H. Mies, L. F. DiMauro, E. Charron, and B. Yang, J. Phys. B 28, 309 (1995).

[6] J. H. Posthumus, Rep. Prog. Phys. 67, 623 (2004).

[7] A. Giusti-Suzor, X. He, O. Atabek, and F. H. Mies, Phys. Rev. Lett. 64, 515 (1990).

[8] L. Y. Peng, I. D. Williams, and J. F. McCann, J. Phys. B 38, 1727 (2005).

[9] P. H. Bucksbaum, A. Zavriyev, H. G. Muller, and D. W. Schumacher, Phys. Rev. Lett. 64, 1883 (1990).

[10] G. Jolicard and O. Atabek, Phys. Rev. A 46, 5845 (1992).

[11] A. Zavriyev, P. H. Bucksbaum, J. Squier, and F. Saline, Phys. Rev. Lett. 70, 1077 (1993).

[12] A. Giusti-Suzor and F. H. Mies, Phys. Rev. Lett. 68, 3869 (1992).

[13] L. J. Frasinski, J. H. Posthumus, J. Plumridge, K. Codling, P. F. Taday, and A. J. Langley, Phys. Rev. Lett. 83, 3625 (1999).

[14] J. H. Posthumus, J. Plumridge, L. J. Frasinski, K. Codling, E. J. Divall, A. J. Langley, and P. F. Taday, J. Phys. B 33, L563 (2000).

[15] L. J. Frasinski, J. Plumridge, J. H. Posthumus, K. Codling, P. F. Taday, E. J. Divall, and A. J. Langley, Phys. Rev. Lett. 86, 2541 (2001).

[16] B. Yang, M. Saeed, L. F. DiMauro, A. Zavriyev, and P. H. Bucksbaum, Phys. Rev. A 44, R1458 (1991).

[17] G. N. Gibson, M. Li, C. Guo, and J. Neira, Phys. Rev. Lett. 79, 2022 (1997).

[18] J. Ludwig, H. Rottke, and W. Sandner, Phys. Rev. A 56, 2168 (1997).

[19] G. H. Dunn, J. Chem. Phys. 44, 2592 (1966).

[20] Z. Amitay, A. Baer, M. Dahan, J. Levin, Z. Vager, D. Zajfman, L. Knoll, M. Lange, D. Schwalm, 
R. Wester, A. Wolf, I. F. Schneider, and A. Suzor-Weiner, Phys. Rev. A 60, 3769 (1999).

[21] P. A. Orr, I. D. Williams, J. B. Greenwood, I. C. E. Turcu, W. A. Bryan, J. Pedregosa-Gutierrez, and C. W. Walter, Phys. Rev. Lett. 98, 163001 (2007).

[22] J. D. Alexander, C. R. Calvert, R. B. King, O. Kelly, W. A. Bryan, G. R. A. J. Nemeth, W. R. Newell, C. A. Froud, I. C. E. Turcu, E. Springate, P.A. Orr, J. Pedregosa-Gutierrez, C. W. Walter, R. A. Williams, I. D. Williams, and J. B. Greenwood, J. Phys. B 42, 154027 (2009).

[23] Z. Amitay, D. Zajfman, and P. Forck, Phys. Rev. A 50, 2304 (1994).

[24] R. Bhattacharya, and S. S. Bhattacharya, Phys. Rev. A 79, 043415 (2009).

[25] B. Dutta, R. Bhattacharya, and S. S. Bhattacharyya, Phys. Rev. A 80, 043413 (2009).

[26] K.-J. Yuan, Z.-T. Liu, J. Yu, M.-D. Chen, and S.-L. Cong, J. Thoer. Comput. Chem. 8, 1197 (2009).

[27] K.-J. Yuan, Z.-T. Liu, and S.-L. Cong J. Mod. Opt. 58, 873 (2011).

[28] S. Chatterjee, B. Dutta, and S. S. Bhattacharyya, Phys. Rev. A 83, 063413 (2011).

[29] D. Yang and S.-L. Cong, Phys. Rev. A 84, 013424 (2011).

[30] R. Bhattacharya, S. Chatterjee, and S. S. Bhattacharya, Phys. Rev. A 85, 033424 (2012).

[31] Z. M. Jia, Z. N. Zeng, R. X. Li, and Z. Z. Xu, J. Phys. B 49, 235401 (2016).

[32] M. F. Kling, Ch. Siedschlag, A. J. Verhoef, J. I. Khan, M. Schultze, Th. Uphues, Y. Ni, M. Uiberacker, M. Drescher, F. Krausz, and M. J. J. Vrakking, Science, 312, 246 (2006).

[33] A. Natan, M. R. Ware, V. S. Prabhudesai, U. Lev, B. D. Bruner, O. Heber, and P. H. Bucksbaum, Phys. Rev. Lett. 116, 143004 (2016).

[34] G. J. Halász, Á. Vibók, N. Moiseyev, and L. S. Cederbaum, Phys. Rev. A 88, 043413 (2013).

[35] J. Wu, M. Kunitski, M. Pitzer, F. Trinter, L. P. H. Schmidt, T. Jahnke, M. Magrakvelidze, C. B. Madsen, L. B. Madsen, U. Thumm, and R. Dörner, Phys. Rev. Lett. 111, 023002 (2013).

[36]] Y.-C. Han and L. B. Madsen, Phys. Rev. A 87, 043404 (2013).

[37] X.-Y. Miao and C.-P. Zhang, Phys. Rev. A 87, 053403 (2013).

[38] X. Gong, P. He, Q. Song, Q. Ji, H. Pan, J. Ding, F. He, H. Zeng, and J. Wu, Phys. Rev. Lett. 113, $203001(2014)$ 
[39] S. Jiang, C. Yu, G. Yuan, T. Wu, and R. Lu, Sci. Rep. 7, 42086 (2017).

[40] B. D. Esry and H. R. Sadeghpour, Phys. Rev. A 60, 3604 (1999).

[41] W. Zhang, C.-C. Shu, T.-S. Ho, H. Rabitz, and S.-L. Cong, J. Chem. Phys. 140, 094304 (2014).

[42] C. C. Marston and G. G. Balint-Kurti, J. Chem. Phys. 91, 3571 (1989).

[43] P. Q. Wang, A. M. Sayler, K. D. Carnes, J. F. Xia, M. A. Smith, B. D. Esry, and I. Ben-Itzhak, Phys. Rev. A 74, 043411 (2006).

[44] P. A. Orr, Ph.D thesis, Queens University Belfast (2007). 

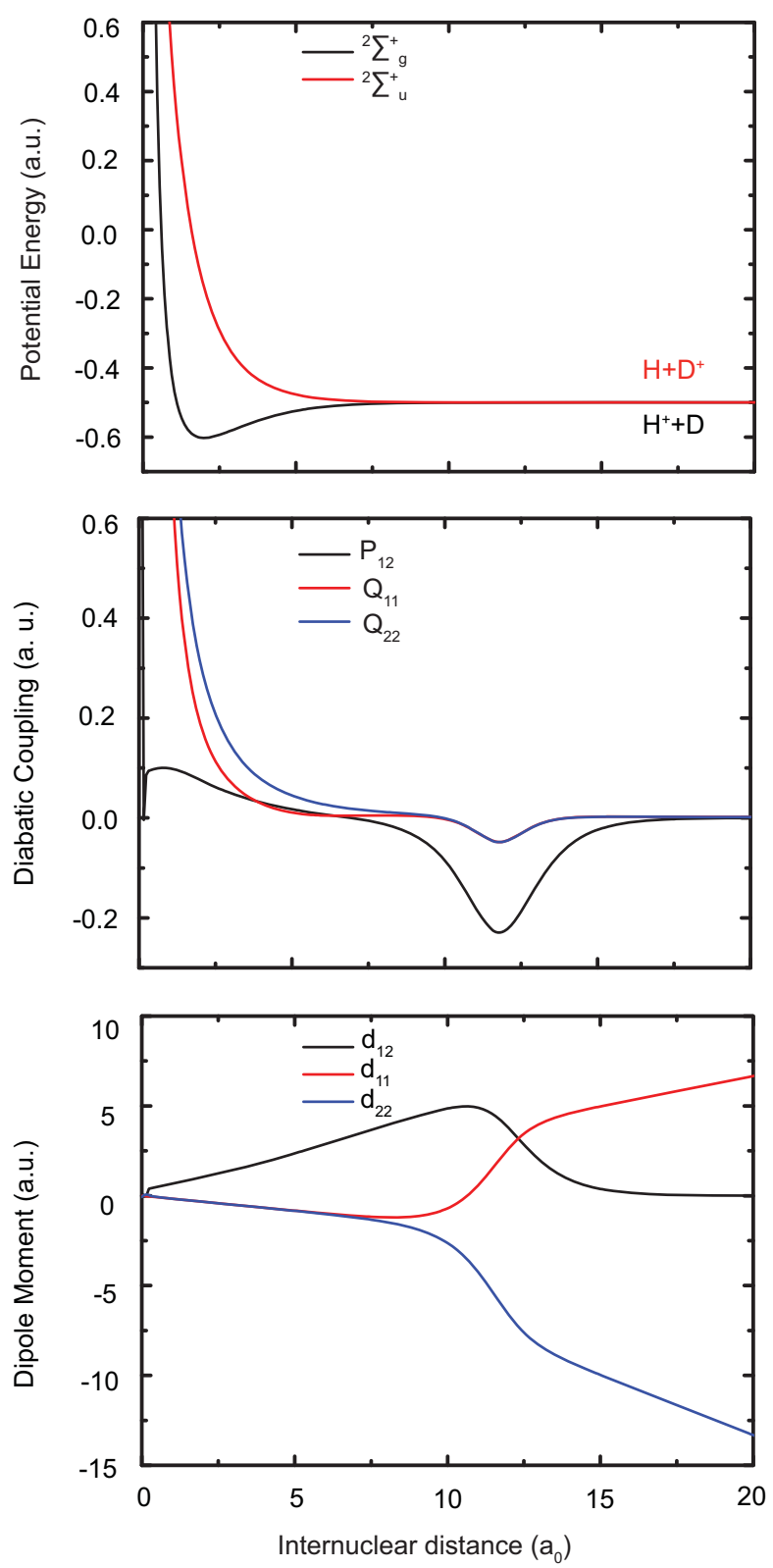

FIG. 1. (a) The adiabatic potential energies of the ${ }^{2} \Sigma_{\mathrm{g}}^{+}\left(1 s \sigma_{\mathrm{g}}\right)$ and ${ }^{2} \Sigma^{+}{ }_{\mathrm{u}}\left(2 p \sigma_{\mathrm{u}}\right)$ states. (b) The nonadiabatic coupling $P_{12}(R), Q_{11}(\mathrm{R})$ and $Q_{22}(\mathrm{R})$ versus internuclear distance. (c) $d_{11}$ and $d_{22}$ denote the permanent dipole moment of ${ }^{2} \Sigma_{\mathrm{g}}^{+}$and ${ }^{2} \Sigma_{\mathrm{u}}^{+}$states. $d_{12}$ displays the transition dipole moment between two electronic states. The relevant molecular data are taken from Ref. [24]. 


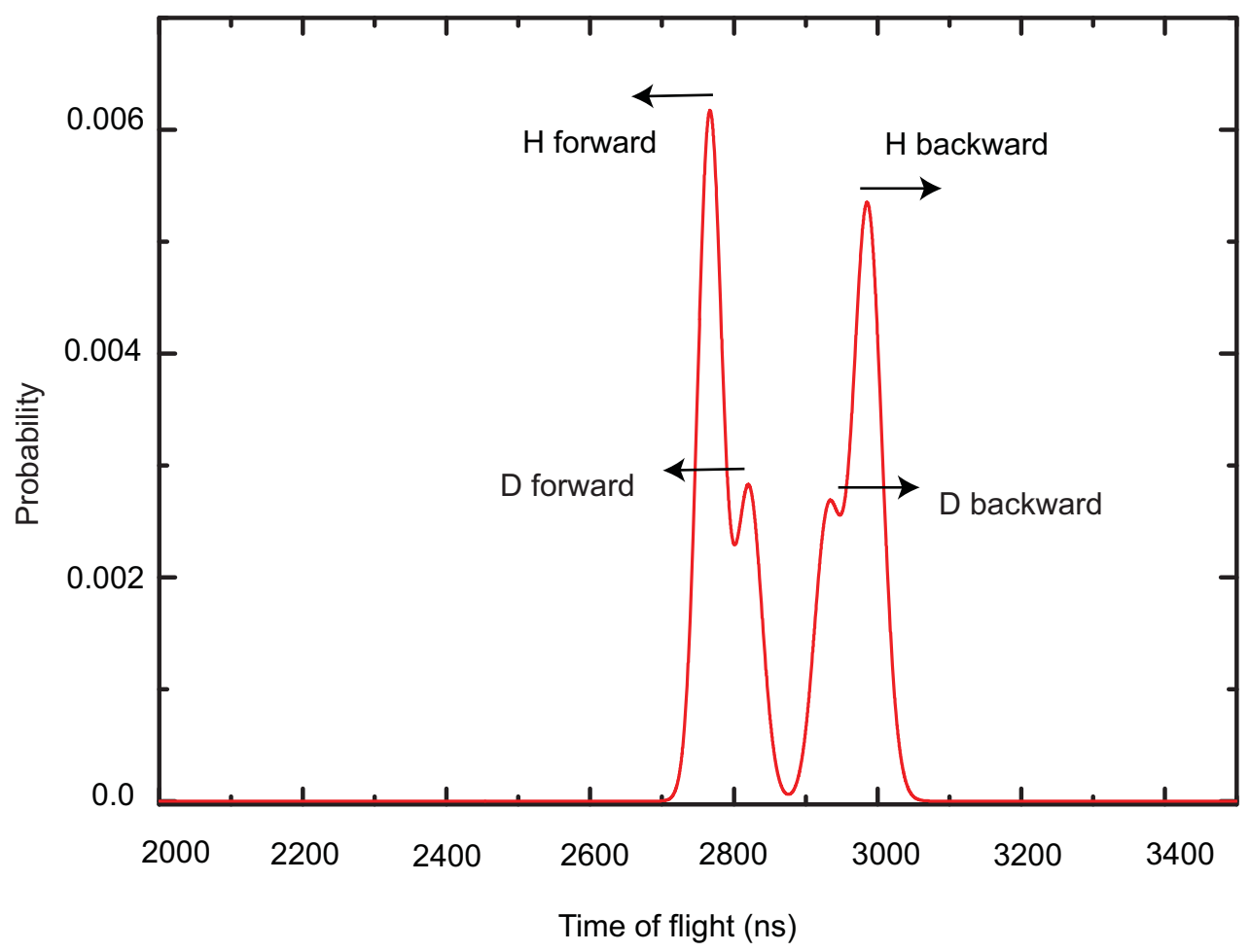

FIG. 2. The theoretical TOF spectrum obtained from an initial vibrational state $v=10$ at a $1 \mathrm{keV}$ energy $\mathrm{HD}^{+}$ion beam at a laser intensity $10^{12} \mathrm{~W} / \mathrm{cm}^{2}$. 


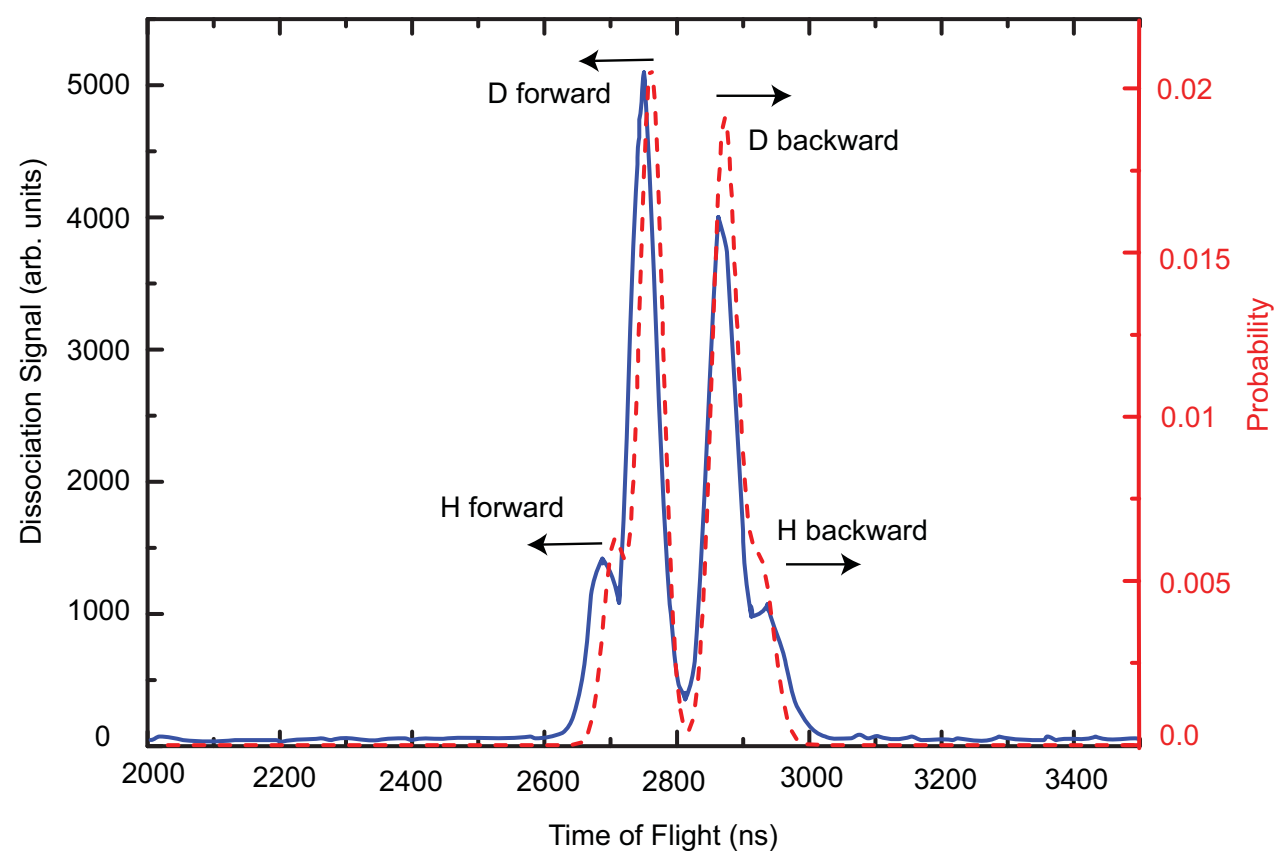

FIG. 3. Comparison of the TOF spectrum in the laboratory frame between experimental results (blue-solid curve) ${ }^{22}$ and our theoretical calculations (red-dashed curve). In theoretical calculations, the initial central kinetic energy of the ion beam was $E=1 \mathrm{keV}$ with an energy spread $\delta E=30 \mathrm{eV}$, a laser intensity $I_{0}=10^{12} \mathrm{~W} / \mathrm{cm}^{2}$ and an initial vibrational state $v=10$. The corresponding theoretical emission probabilities are shown in red font on the right. 


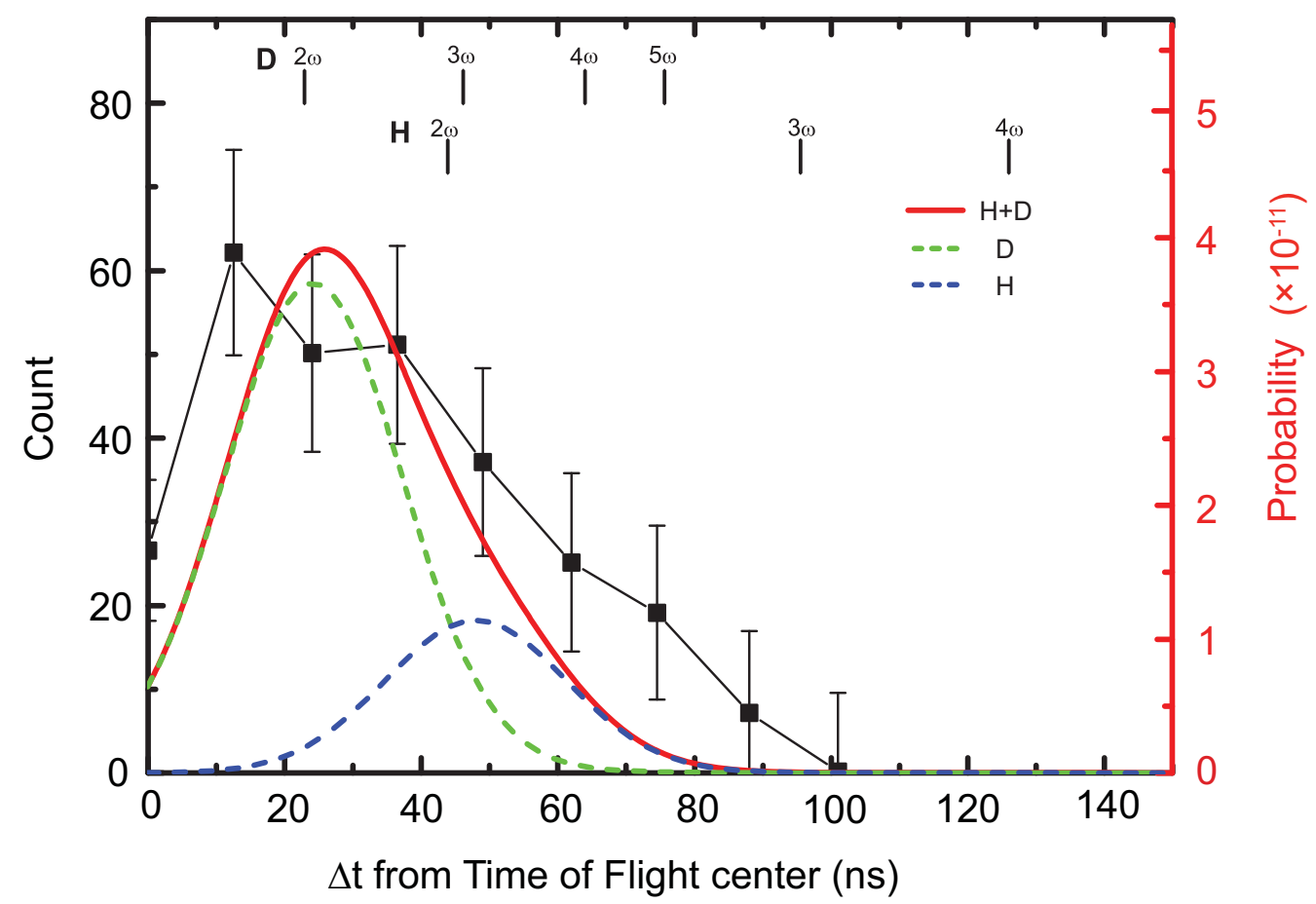

FIG. 4. The red solid line indicates the TOF spectrum in center-of-mass frame arising from $\mathrm{H}$ and D fragments in the theoretical simulation. The data points indicate the TOF spectrum in the center-of-mass frame from experimental measurements, ${ }^{21}$ where the vertical error bars indicate statistical uncertainties at the one sigma level. Vertical lines mark the expected positions of $\mathrm{H}$ and $\mathrm{D}$ fragments due to absorption of an integer number of photons by the molecular ion $\mathrm{HD}^{+}$. In the theoretical calculations, the initial central kinetic energy of the ion beam was $E=2 \mathrm{keV}$ with an energy spread $\delta E=60 \mathrm{eV}$, a laser intensity of $I_{0}=5 \times 10^{12} \mathrm{~W} / \mathrm{cm}^{2}$ and for an initial vibrational state $v=0$. 


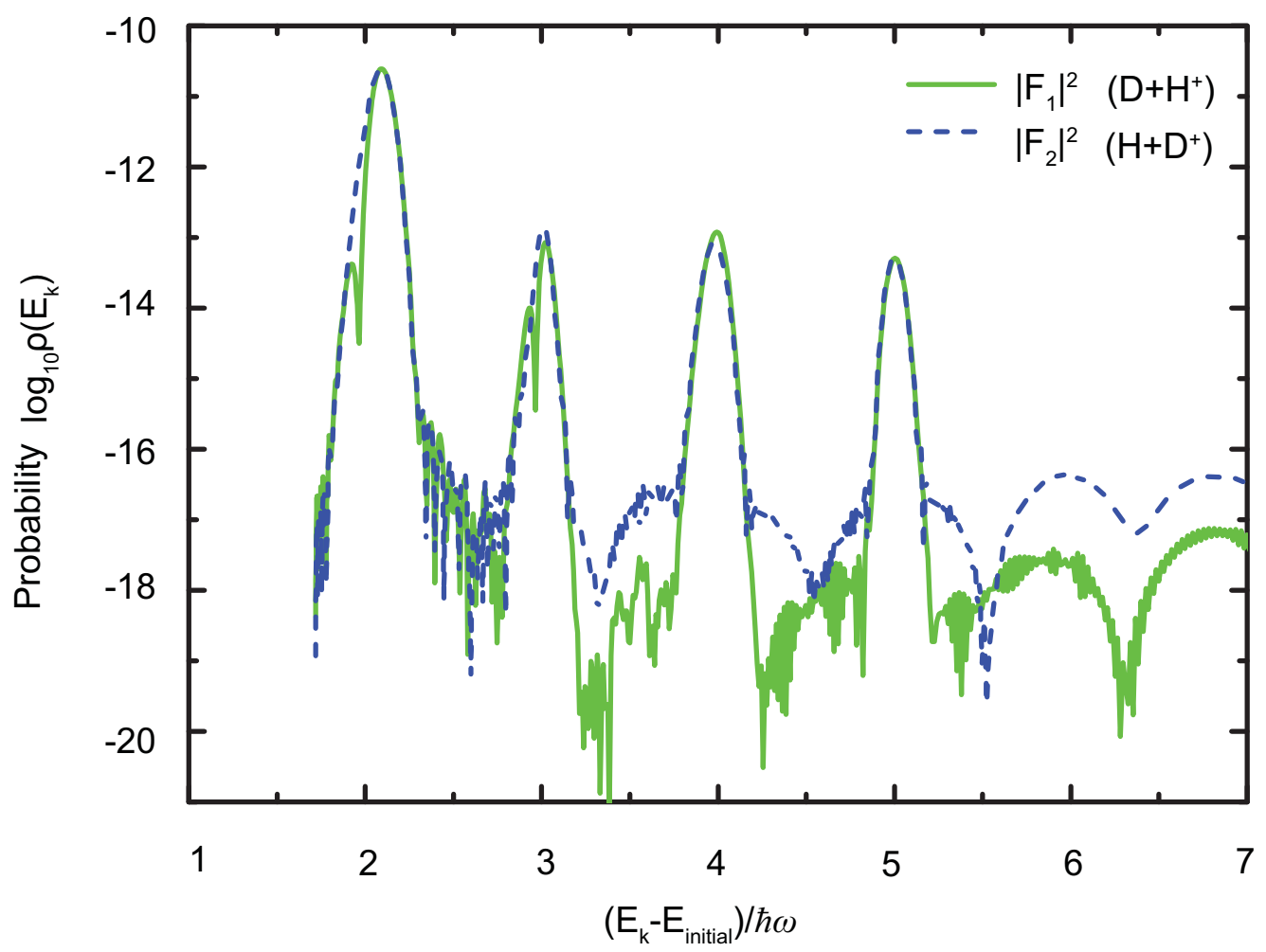

FIG. 5. The kinetic energy distribution of dissociation fragments from initial vibrational state $v=$ 0 with laser intensity $I_{0}=5 \times 10^{12} \mathrm{~W} / \mathrm{cm}^{2}$. The solid and dashed curves show the kinetic energy distributions from channel $1 s \sigma_{g}$ and channel $2 p \sigma_{u}$, respectively. The tick labels on the horizontal axis indicates the energy difference between the initial vibrational level $(v=0)$ and the kinetic energy of the dissociated fragments. 

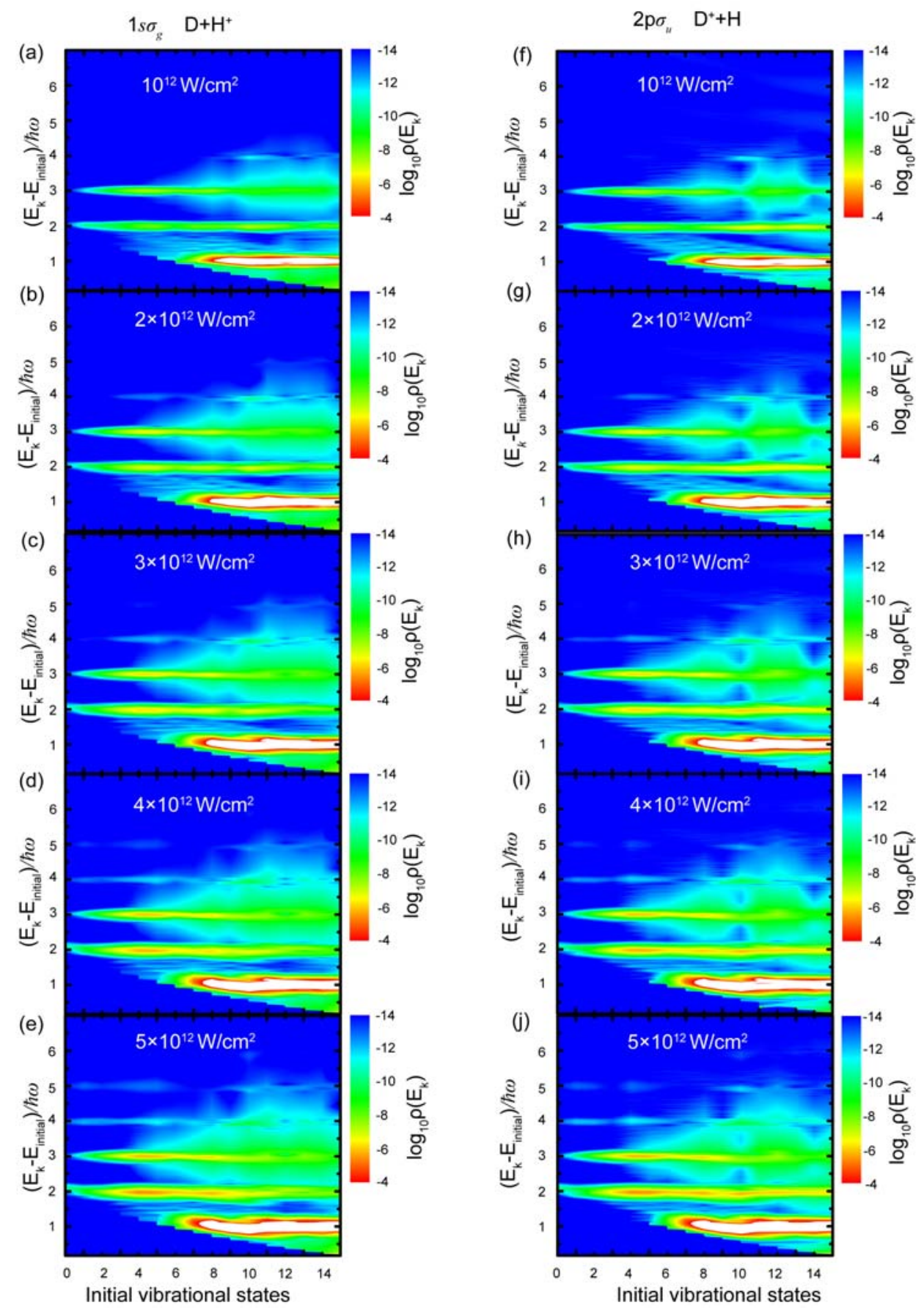

FIG. 6. The relative kinetic energy distributions of dissociated fragments from different initial vibrational states at a variety of laser intensities. Panels (a)-(e) denote kinetic energy distributions from channel $1 s \sigma_{g}$ with the laser intensity from $10^{12}$ to $5 \times 10^{12} \mathrm{~W} / \mathrm{cm}^{2}$. Panels (f)-(j) denote the corresponding dissociation from channel $2 p \sigma_{u}$. 

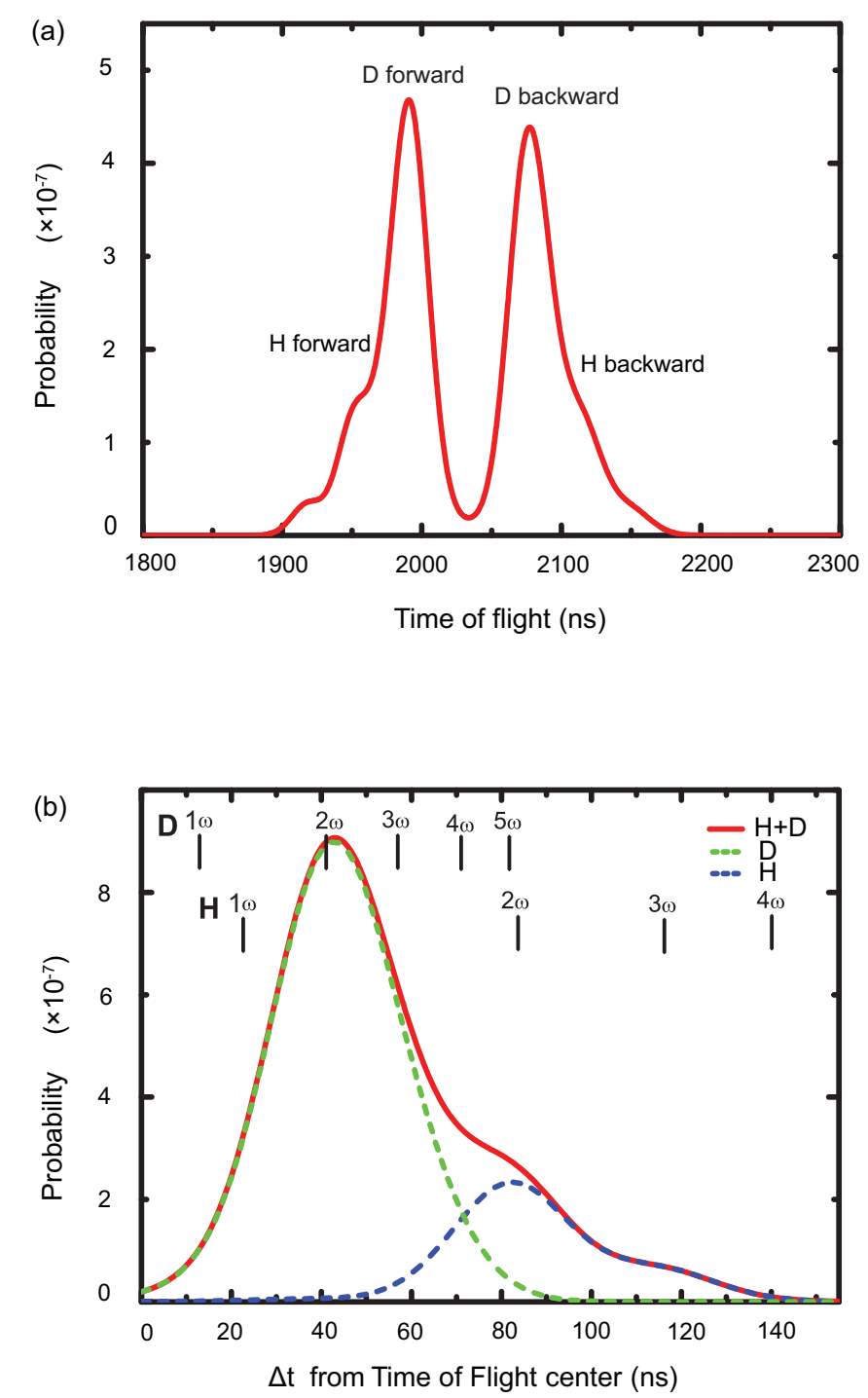

FIG. 7. The TOF spectrum starting from the vibrational state $v=6$ at a laser intensity of $I_{0}=5 \times$ $10^{12} \mathrm{~W} / \mathrm{cm}^{2}$. (a) The TOF spectrum of neutral fragments in the laboratory frame. (b) The green and blue dash curves indicate the TOF spectra of center-of-mass frame of D and H fragments, respectively. The red curve indicates the sum of the $\mathrm{H}$ and $\mathrm{D}$ components. 


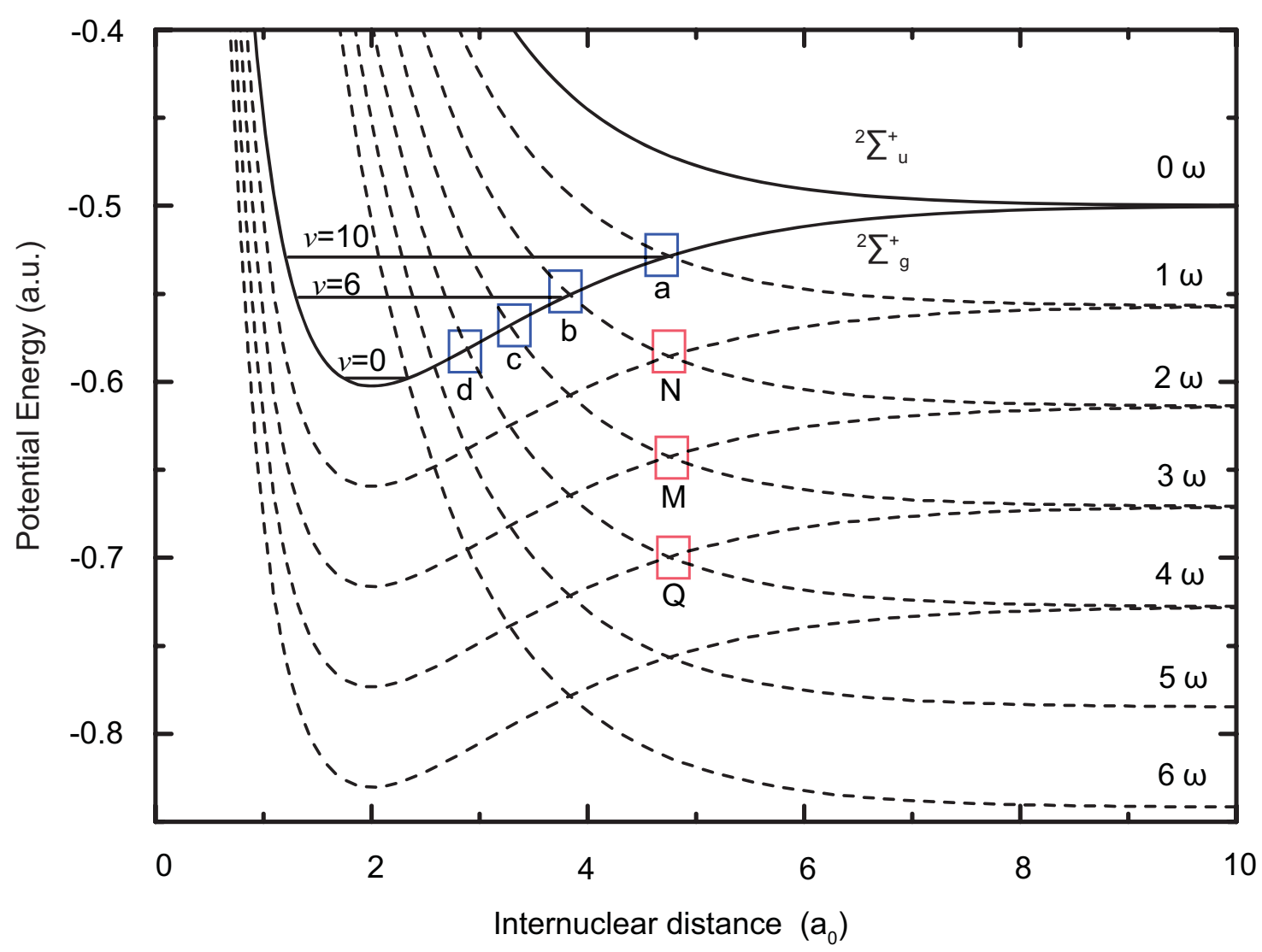

FIG. 8. Diabatic dressed potential energy curves for $\mathrm{HD}^{+}$in the laser field of wavelength $800 \mathrm{~nm}$.

The horizontal solid lines denote the vibrational energy levels $v=0,6$, and 10 of the ground electronic state ${ }^{2} \Sigma_{\mathrm{g}}^{+}\left(1 s \sigma_{g}\right)$. The blue squares labeled as a, b, c and d, denote the diabatic crossing points of the field-free ground electronic state ${ }^{2} \Sigma^{+}{ }_{\mathrm{g}}\left(1 s \sigma_{\mathrm{g}}\right)$ with the first excited repulsive state ${ }^{2} \Sigma_{\mathrm{u}}^{+}$ $\left(2 p \sigma_{u}\right)$ dressed by $1,2,3$ and 4 photons, respectively. 

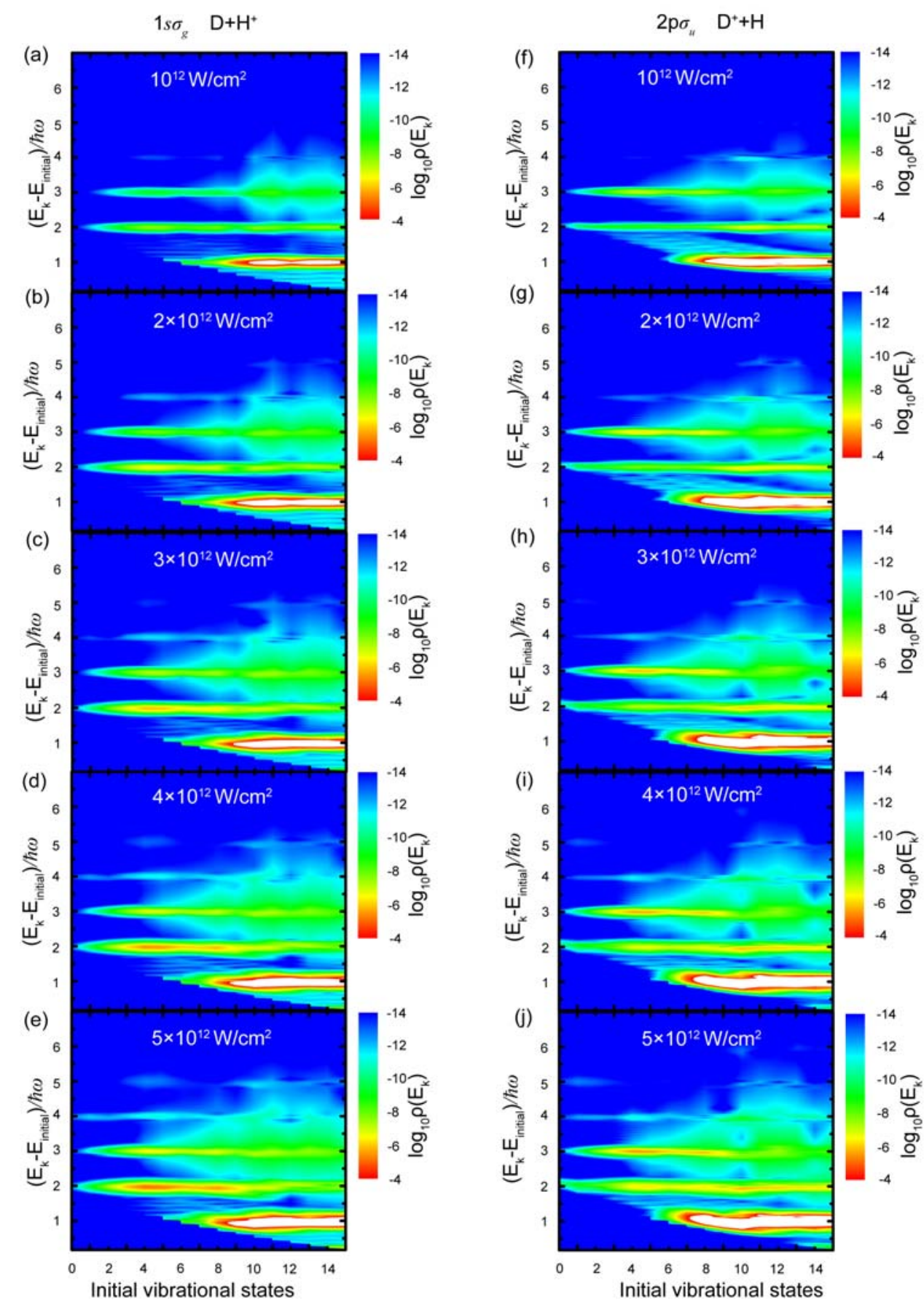

FIG. 9. Same as Fig. 6 except that the nonadiabatic coupling terms, $P_{i j}$ and $Q_{i j}$ in Eq. (1), are set to be zero. 


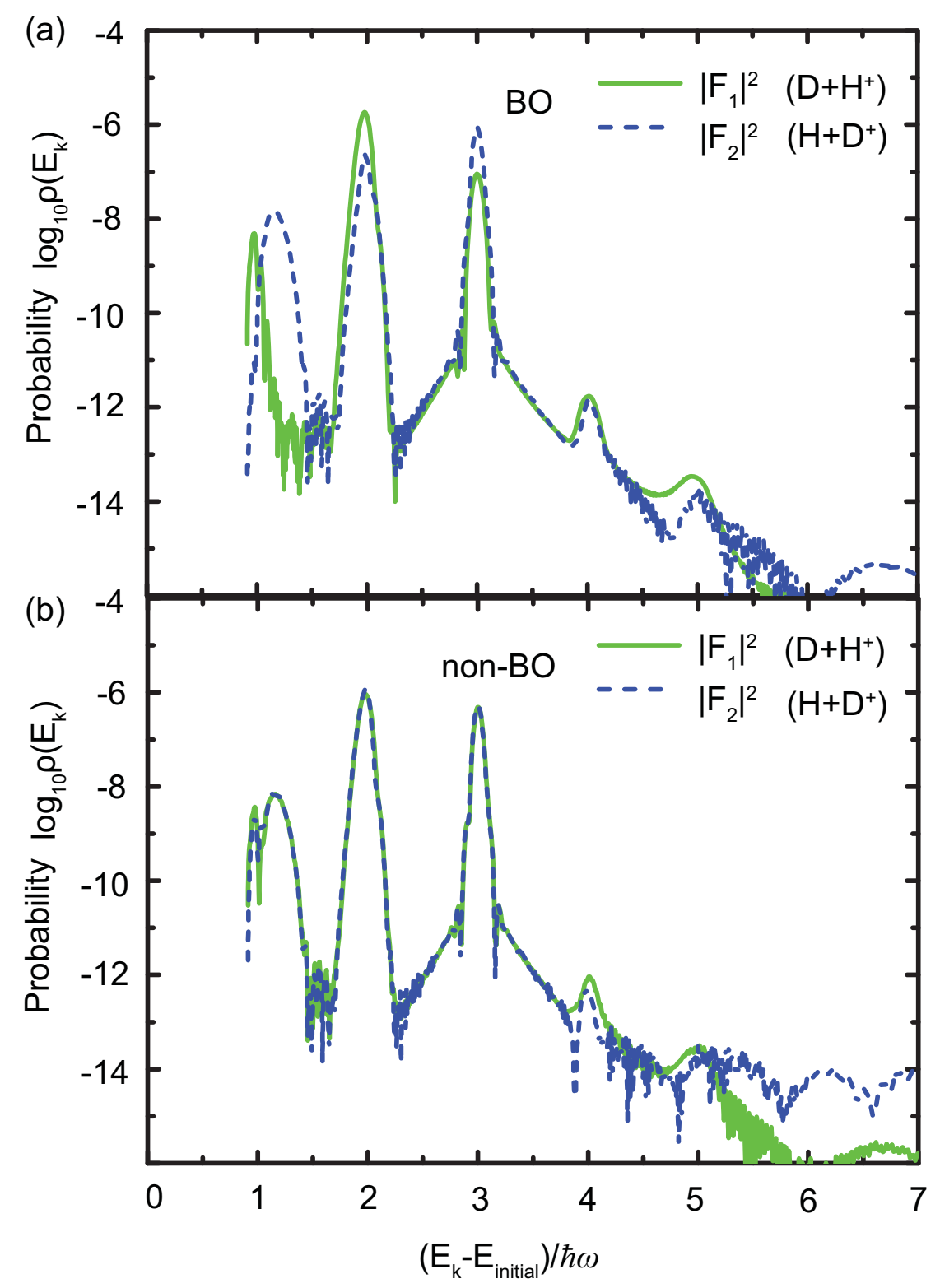

FIG. 10. The kinetic energy distribution of dissociation fragments for initial vibrational state $v=6$ at the laser intensity $I_{0}=5 \times 10^{12} \mathrm{~W} / \mathrm{cm}^{2}$. (a) The nonadiabatic couplings are neglected; (b) the nonadiabatic couplings are included. 


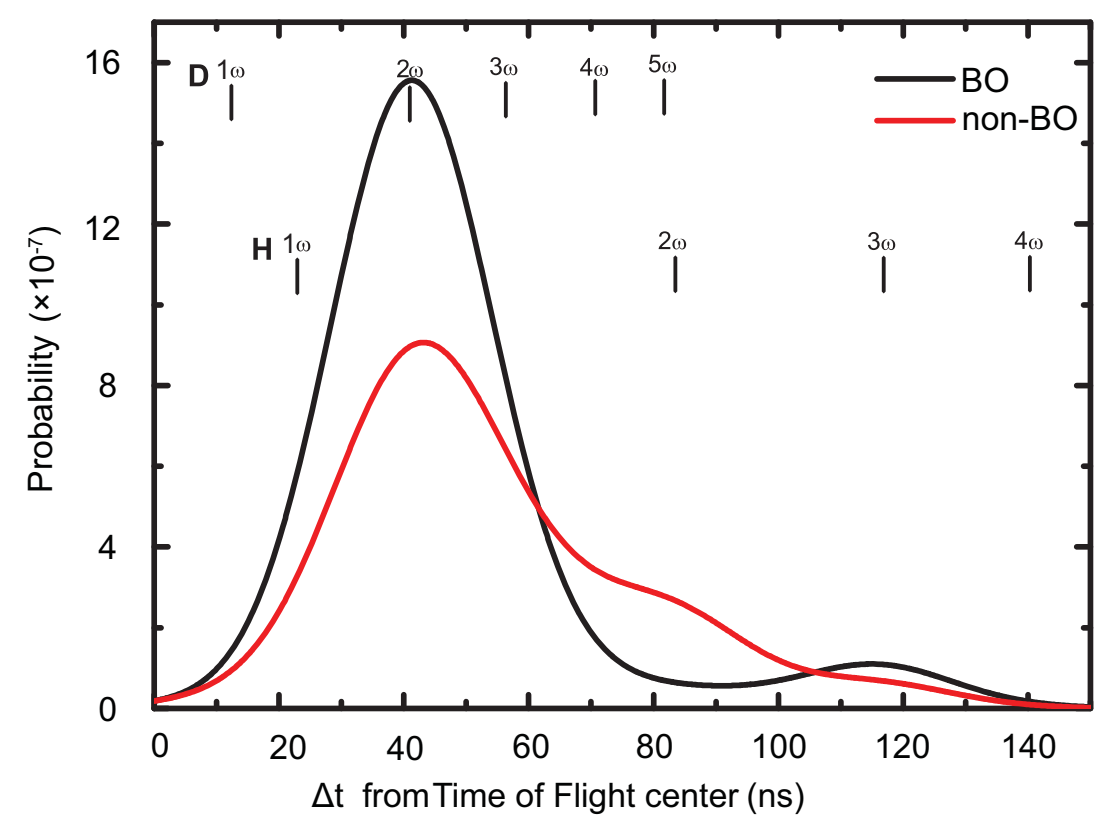

FIG. 11. The TOF spectra in center-of-mass frame for initial vibrational state $v=6$ at the laser intensity $I_{0}=5 \times 10^{12} \mathrm{~W} / \mathrm{cm}^{2}$. The black curve denotes the calculation with the nonadiabatic couplings turned off (labeled as BO). For comparison, the red curve, which is the same as the one in Fig. 7(b), denotes the calculation with consideration of the nonadiabatic couplings (labeled as non-BO). 